\title{
Emergent Adler-Bardeen theorem
}

\author{
Vieri Mastropietro \\ Department of Mathematics "F. Enriquez", University of Milano, \\ Via C. Saldini 50, 20133 Milano, Italy \\ E-mail: vieri.mastropietro@unimi.it
}

ABSTRACT: We consider a $\mathrm{QED}_{d+1}, d=1,3$ lattice model with emergent Lorentz or chiral symmetry, both when the interaction is irrelevant or marginal. While the correlations present symmetry breaking corrections, we prove that the Adler-Bardeen (AB) non-renormalization property holds at a non-perturbative level even at finite lattice: all radiative corrections to the anomaly are vanishing. The analysis uses a new technique based on the combination of non-perturbative regularity properties obtained by exact renormalization Group methods and Ward Identities. The AB property, essential for the renormalizability of the standard model, is therefore a robust feature imposing no constraints on possible symmetry breaking terms, at least in the class of lattice models considered.

Keywords: Anomalies in Field and String Theories, Lattice Quantum Field Theory, Nonperturbative Effects, Renormalization Group

ARXIV EPRINT: 2001.00183 


\section{Contents}

1 Introduction 1

2 Lattice models and anomaly non-renormalization 2

$\begin{array}{lll}3 & \text { Renormalization Group } & 7\end{array}$

4 Anomaly non-renormalization; the irrelevant case $\quad 10$

5 Anomaly non-renormalization; marginal interactions 13

6 Conclusions $\quad 15$

\section{Introduction}

According to modern understanding several symmetries of particle physics can be approximate and emergent, see e.g. [1-3], and possibly broken at the Planck length scale. The Adler-Bardeen $(\mathrm{AB})$ non-renormalization property [4-6] is essential to ensure the renormalizability of the Standard model, through the anomaly cancellation. The proof of the $\mathrm{AB}$ property is based on identities between class of graphs and relies on the validity of Lorentz and chiral symmetry. If the symmetry breaking terms are dimensionally irrelevant, one expects that the corrections are of the order of the Planck divided by the particle physics length scale, hence typically undetectable. However this would be not true if corrections are present to the $\mathrm{AB}$ property; even very small radiative contributions would be amplified if they break renormalizability. It is therefore interesting to see if the anomaly non-renormalization holds generically even when symmetry breaking terms are present at the Planck length scale, or if in contrast its validity requires that they are absent or at least of special form.

We consider the simple situation where the symmetry violation is produced by a lattice, with spacing small compared to particle physics lengths but large or comparable to the Planck length scale; lattice models are often used to mimic the violation of symmetries, see e.g. [7-10]. In particular, the model we consider is the interacting extension of the Nielsen-Ninomiya simulation of the chiral anomaly [11], that is lattice fermions coupled with a quantum massive photon field, with an emerging description in terms of massless $\mathrm{QED}_{d+1}, d=1,3$. There are corrections to the Lorentz invariant part of the correlations which are non-vanishing and of order of the momentum times the lattice spacing. In contrast, we prove that the anomaly is perfectly non-renormalized, even in presence of finite symmetry breaking terms; that is, at least in the class of lattice models we consider, the $A B$ non-renormalization is a robust feature imposing no constraints on the symmetry breaking terms. 
Our results are based on a novel technique based on the combination of constructive regularity properties obtained by exact Renormalization Group (RG) methods and Ward Identities. The contribution of irrelevant terms at each step is essential and fully taken into account. The results are fully non-perturbative, as physical quantities are expressed in terms of series whose convergence is established in presence of a finite photon mass, as consequence of cancellations due to Pauli principle (see [12] for an introduction to such methods). This is a major difference with respect to other approaches to the anomaly which give results valid only order by order, see e.g. [13, 14]. The strategy of proof was used in [15] for irrelevant interactions and is here extended to the marginal case. Even if the validity of the $\mathrm{AB}$ property is proved for the $\mathrm{U}(1)$ gauge group and with a photon mass regularization, the result indicates that the anomaly cancellation condition is common between the continuum theory and the lattice theory and suggests that anomaly-free chiral gauge theory, as the Standard model, can be formulated non-perturbatively by lattice formulation.

\section{Lattice models and anomaly non-renormalization}

The massless lattice $\mathrm{QED}_{d+1}$ model we consider is the interacting extension of the NielsenNinomiya anomaly simulation [11], where the interaction with a quantum photon field is included. The detailed form of the lattice has no importance and we do a specific choice just for definiteness.

In $d=1$ we consider a linear lattice $\Lambda=\{x=n a, n \in \mathbb{Z}\}$ ). If $\psi_{x}^{ \pm}, x \in \Lambda$, are fermionic creation or annihilation operators defined on the Fock space and verifying $\left\{\psi_{x}^{+}, \psi_{y}^{-}\right\}=\delta_{x, y}$, $\left\{\psi_{x}^{+}, \psi_{y}^{+}\right\}=\left\{\psi_{x}^{-}, \psi_{y}^{-}\right\}=0$, the lattice Hamiltonian is

$$
H_{0}=\frac{t}{a} \sum_{x}\left(\frac{1}{2}\left(\psi_{x+a}^{+} \psi_{x}^{-}+\psi_{x}^{+} \psi_{x+a}^{-}\right)-\cos (\zeta) \psi_{x}^{+} \psi_{x}^{-}\right)
$$

We define

$$
\widehat{\psi}_{k}^{ \pm}=\sum_{x \in \Lambda} e^{\mp i k x} \psi_{x}^{ \pm} \quad \psi_{x}^{ \pm}=\int \frac{d k}{(2 \pi)} e^{ \pm i k x} \widehat{\psi}_{k}^{ \pm}
$$

where $|k| \leq \pi / a$ and

$$
H_{0}=\int \frac{d k}{(2 \pi)} h(k) \widehat{\psi}_{k}^{+} \widehat{\psi}_{k}^{-} \quad h(k)=\frac{t}{a}(\cos k a-\cos \zeta)
$$

Note that for $k= \pm \zeta / a+q$ one has $h(k)= \pm v q+O\left(q^{2} a\right), v=t \sin \zeta$, that is the dispersion relation is essentially linear.

In $d=3$ we consider a lattice formed by two sublattices: one is $\Lambda_{1}=\left\{n_{1} a\left(\frac{1}{\sqrt{2}}, \frac{1}{\sqrt{2}}, 0\right)+\right.$ $\left.n_{2} a\left(-\frac{1}{\sqrt{2}}, \frac{1}{\sqrt{2}}, 0\right)+n_{3} a(0,0,1), n_{i} \in \mathbb{Z}\right\}$, and the other is $\Lambda_{2}=\Lambda_{1}+a \frac{1}{\sqrt{2}} e_{1} \equiv \Lambda_{1}+a \delta_{1}$ (we let $e_{1}, e_{2}, e_{3}$ be the elements of the standard Euclidean basis and $\delta_{1}=\frac{1}{\sqrt{2}} e_{1}, \delta_{2}=\frac{1}{\sqrt{2}} e_{2}$ ). We associate a fermionic field to each sublattice $\psi_{x, j}^{ \pm}$with $x \in \Lambda_{j}, j=1,2$ and we consider 
the Hamiltonian

$$
\begin{aligned}
H_{0}= & -\frac{t}{2 a} \sum_{x \in \Lambda_{1}}\left[\left(i \psi_{x, 1}^{+} \psi_{x+a \delta_{1}, 2}^{-}+i \psi_{x-a \delta_{1}, 2}^{+} \psi_{x, 1}^{-}+c . c .\right)+\left(\psi_{x, 1}^{+} \psi_{x+a \delta_{2}, 2}^{-}-\psi_{x-a \delta_{2}, 2}^{+} \psi_{x, 1}^{-}+c . c .\right)\right]- \\
& t^{\prime} \sum_{x \in \Lambda_{1}}\left[\frac{1}{2 a}\left(\psi_{x, 1}^{+} \psi_{x+a e_{3}, 1}^{-}+\psi_{x, 1}^{+} \psi_{x-a e_{3}, 1}^{-}\right)-\left(\cos (\zeta)-\frac{1}{a}\right) \psi_{x, 1}^{+} \psi_{x, 1}^{-}\right]+ \\
& t^{\prime} \sum_{x \in \Lambda_{2}}\left[\frac{1}{2 a}\left(\psi_{x, 2}^{+} \psi_{x+a e_{3}, 2}^{-}+\psi_{x, 2}^{+} \psi_{x-a e_{3}, 2}^{-}\right)-\left(\cos (\zeta)-\frac{1}{a}\right) \psi_{x, 2}^{+} \psi_{x, 2}^{-}\right]+ \\
& \frac{t^{\prime}}{4 a} \sum_{x \in \Lambda_{1}} \psi_{x, 1}^{+}\left(\psi_{x+a \delta_{1}+a \delta_{2}, 1}^{-}+\psi_{x+a \delta_{1}-a \delta_{2}, 1}^{-}+\psi_{x-a \delta_{1}+a \delta_{2}, 1}^{-}+\psi_{x-a \delta_{1}-a \delta_{2}, 1}^{-}\right)- \\
& \frac{t^{\prime}}{4 a} \sum_{x \in \Lambda_{2}} \psi_{x, 2}^{+}\left(\psi_{x+a \delta_{1}+a \delta_{2}, 2}^{-}+\psi_{x+a \delta_{1}-a \delta_{2}, 2}^{-}+\psi_{x-a \delta_{1}+a \delta_{2}, 2}^{-}+\psi_{x-a \delta_{1}-a \delta_{2}, 2}^{-}\right)
\end{aligned}
$$

We pass to Fourier space:

$$
\psi_{x, 1}^{ \pm}=\int \frac{d k}{(2 \pi)^{3}} e^{ \pm i k x} \widehat{\psi}_{k, 1}^{ \pm} \quad \psi_{x, 2}^{ \pm}=\int \frac{d k}{(2 \pi)^{3}} e^{ \pm i k x} \widehat{\psi}_{k, 2}^{ \pm}
$$

where the integrals are over the first Brillouin zone so that the Hamiltonian reads:

$$
H_{0}=\int \frac{d k}{(2 \pi)^{3}} \widehat{\psi}_{k}^{+}\left(\begin{array}{cc}
\alpha(k) & \beta(k) \\
\beta^{*}(k) & -\alpha(k)
\end{array}\right) \widehat{\psi}_{k}^{-} \equiv \int \frac{d k}{(2 \pi)^{3}} \widehat{\psi}_{k}^{+} h(k) \widehat{\psi}_{k}^{-},
$$

where

$$
\begin{aligned}
\alpha(k) & =-\frac{t^{\prime}}{a}\left(\cos k_{3} a-\cos \zeta\right)+\frac{t^{\prime}}{a}\left(\cos \frac{a k_{1}}{\sqrt{2}} \cos \frac{a k_{2}}{\sqrt{2}}-1\right), \\
\beta(k) & =-\frac{t}{a} \sin \frac{k_{1}}{\sqrt{2}}+i \frac{t}{a} \sin \frac{k_{2}}{\sqrt{2}} .
\end{aligned}
$$

If $k_{1}=q_{1}, k_{2}=q_{2}$ and $k_{3}= \pm \zeta / a+q_{3}$ we can write $h(k)=-v_{1} \sigma_{1} q_{1}-v_{1} \sigma_{2} q_{2} \mp v \sigma_{3} q_{3}+O\left(a|q|^{2}\right)$ with $v_{1}=t / \sqrt{2}, v=t^{\prime} \sin \zeta$ and $q=\left(q_{1}, q_{2}, q_{3}\right)$. We denote by $\widehat{\psi}_{x_{0}, k, i}=e^{H_{0} x_{0}} \widehat{\psi}_{k, i}^{ \pm} e^{-H_{0} x_{0}}$ where $x_{0}$ is the Euclidean time; we introduce the 2-point function $\widehat{g}_{i, j}(\mathbf{k}) \equiv<\widehat{\psi}_{\mathbf{k}, i}^{+} \widehat{\psi}_{\mathbf{k}, j}^{-}>$given by the following matrix

$$
\widehat{g}(\mathbf{k})=\frac{1}{-i k_{0} I+h(k)}
$$

where $\mathbf{k}=\left(k_{0}, k\right)$. A similar expression holds in $d=1$. The Fourier transform of the propagator $\widehat{g}(\mathbf{k})$ is denoted by $g(\mathbf{x})$ with $\mathbf{x}=\left(x_{0}, x\right)$.

It is well known that the above lattice models admit an emerging description in terms of Dirac particles [11]. Indeed a Dirac massless particle has propagator $\left\langle\bar{\Psi}_{\mathbf{k}} \Psi_{\mathbf{k}}\right\rangle=\frac{1}{-i \mathbf{k}}$, where $\mathrm{k}=\gamma_{\mu} k_{\mu}, \mu=0,1, \ldots d$ and with $\left\{\gamma_{\mu}, \gamma_{\nu}\right\}=2 \delta_{\mu, \nu}$ and the Euclidean Dirac derivative is $\not \partial$. In $d=1$ a possible realization of $\gamma$-matrices is $\sigma_{1}=\gamma_{0}, \sigma_{2}=\gamma_{1}$ and $\sigma_{3}=\gamma_{5}$ with $\sigma_{1}=\left(\begin{array}{ll}0 & 1 \\ 1 & 0\end{array}\right), \sigma_{2}=\left(\begin{array}{cc}0 & -i \\ i & 0\end{array}\right), \sigma_{3}=\left(\begin{array}{cc}1 & 0 \\ 0 & -1\end{array}\right)$. Similarly in $d=3$
$\gamma_{0}=\left(\begin{array}{ll}0 & I \\ I & 0\end{array}\right) \quad \gamma_{j}=\left(\begin{array}{cc}0 & i \sigma_{j} \\ -i \sigma_{j} & 0\end{array}\right), \quad \gamma_{5}=\left(\begin{array}{cc}I & 0 \\ 0 & -I\end{array}\right)$ 
In addition to Lorentz invariance, Dirac particles verify gauge and chiral symmetry, implying the conservations of the $d+1$ current $J_{\mu}=\bar{\Psi} \gamma_{\mu} \Psi$ and axial current $J_{\mu}=\bar{\Psi} \gamma_{\mu} \gamma_{5} \Psi$. It is also convenient to write $\Psi=\left(\Psi_{+}, \Psi_{-}\right)$and $\bar{\Psi}=\Psi^{+} \gamma_{0}$, so that the Dirac propagator can be written as, $\widehat{g}_{ \pm}^{D}(\mathbf{k})=<\Psi_{ \pm, \mathbf{k}}^{+} \Psi_{ \pm, \mathbf{k}}>$

$$
\widehat{g}_{ \pm}^{D}(\mathbf{k})=\left(-i k_{0} \pm k\right)^{-1} \quad \widehat{g}_{ \pm}^{D}(\mathbf{k})=\left(-i k_{0} \mp \sigma_{1} k_{1} \mp \sigma_{2} k_{2} \mp \sigma_{3} k_{3}\right)^{-1}
$$

in $d=1$ and $d=3$ respectively.

Let us look at the lattice propagator (2.9) restricting to momenta close to $\pm \zeta / a$. We introduce a smooth compact support function $\chi_{\omega}(\mathbf{k})$, with $\omega= \pm$ non vanishing only for $|\mathbf{k}-\omega \bar{\zeta} / a| \leq 1 /(10 a)$ with $\bar{\zeta}=(0, \zeta)$ in $d=1$ or $\bar{\zeta}=(0,0,0, \zeta)$ in $d=3$. In $d=1$ we define $\widehat{g}_{\omega}(\mathbf{k}-\omega \bar{\zeta} / a)=\chi_{\omega}(\mathbf{k}) \widehat{g}(\mathbf{k})$ and in $d=3$ we define

$$
\widehat{g}_{i, j ; \omega}(\mathbf{k}-\omega \bar{\zeta} / a)=\delta_{i, j, \omega} \chi_{\omega}(\mathbf{k}) \widehat{g}_{i, j}(\mathbf{k})
$$

with $\delta_{i, j,+}=1, \delta_{i, j,-}=(-1)^{i+j}$. The function $\widehat{g}_{\omega}$ is the propagator restricted to momenta around $\pm \bar{\zeta} / a$ and, setting $v=v_{1}=1$ we get, calling $\mathbf{k}-\omega \bar{\zeta} / a=\mathbf{q}$

$$
\widehat{g}_{ \pm}(\mathbf{q})=\widehat{g}_{ \pm}^{D}(\mathbf{q})\left(1+r_{ \pm}(\mathbf{q})\right) \quad\left|r_{ \pm}(\mathbf{q})\right| \leq C a|\mathbf{q}|
$$

The lattice models (2.1) and (2.4) admit therefore an emerging description in terms of massless Dirac partcles; the propagator for momenta far from the inverse spacing has a Lorentz invariant part up to corrections which are small but non vanishing. Let us see what happens to the conservation of the currents. The current in a lattice theory can be introduced using the Peierls substitution. In $d=1$ one introduces an interaction with an external gauge field by writing

$$
H_{0}(A)=\frac{t}{a} \sum_{x}\left(\frac{1}{2}\left(\psi_{x+a}^{+} e^{i \int_{x+a}^{x} d s A(s)} \psi_{x}^{-}+\psi_{x}^{+} e^{i \int_{x}^{x+a} d s A(s)} \psi_{x+a}^{-}\right)-\cos (\zeta) \psi_{x}^{+} \psi_{x}^{-}\right)
$$

with a similar expression holding in $d=3$; the current is defined as $j_{x}=\left.\frac{\partial H_{0}(A)}{\partial A(x)}\right|_{A(x)=0}$, and the lattice density is $\rho_{x}=\psi_{x}^{+} \psi_{x}^{-}$, and they can be combined in $j_{\mu}=\left(\rho, j_{1}, \ldots, j_{d}\right)$. The lattice density and current vertex are close, in the sense of correlations and up to corrections as in (2.12), to the Dirac ones $\bar{\Psi} \gamma_{\mu} \Psi$. Such corrections however do not prevent the conservation of the lattice current in the sense of Ward Identities (see (2.26) below), as the Peierls substitution ensures gauge invariance at a lattice level.

A different situation is encountered in the case of chiral currents. Following [11] one can indeed define an analogue of the chiral density and current in the lattice model, by the requirement that it is close to the Dirac chiral current $\bar{\Psi} \gamma_{\mu} \gamma_{5} \Psi$ in the sense of correlations, up to corrections. The lattice chiral density can be defined as the difference of densities of fermions around $\pm \bar{\zeta} / a$, that is in $d=1$

$$
\widehat{\rho}_{p}^{5}=\int \frac{d k}{(2 \pi)} \frac{\sin k a}{\sin \zeta} \widehat{\psi}_{k+p}^{+} \widehat{\psi}_{k}^{-}
$$

or $\widehat{\rho}_{p}^{5}=\int \frac{d k}{(2 \pi)^{3}} \frac{\sin k_{3} a}{\sin \zeta} \widehat{\psi}_{k+p}^{+} \widehat{\psi}_{k}^{-}$; in coordinate space $\rho_{x}^{5}=-\frac{i}{2 \sin \zeta}\left(\psi_{x}^{+} \psi_{x+a e_{3}}^{-}-\psi_{x}^{+} \psi_{x-a e_{3}}^{-}\right)$or $\rho_{x}^{5}=-\frac{i}{2 \sin \zeta}\left(\psi_{x}^{+} \psi_{x+a}^{-}-\psi_{x}^{+} \psi_{x-a}^{-}\right)$. The definition of the axial current is given in a similar 
way inserting a factor $\sin k a$ or $\sin k_{3} a$ in the Fourier transform of the current. The axial symmetry is however broken and there is no conservation of axial current.

Let us introduce now a dynamical photon field $\bar{A}_{\mu}(\mathbf{x})$ (not to be confused with the external field $\left.A_{\mu}\right)$ with integration $P(d \bar{A})$ and propagator

$$
v_{\mu, \nu}(\mathbf{x})=\delta_{\mu, \nu} v(\mathbf{x})=\int \frac{d \mathbf{k}}{(2 \pi)^{d+1}} \chi(\mathbf{k}) \frac{e^{i \mathbf{k x}}}{\mathbf{k}^{2}+M_{a}^{2}} \delta_{\mu, \nu}
$$

where $\mathbf{x}=\left(x_{0}, x\right), \chi(\mathbf{k})$ is a cut-off function vanishing for momenta larger than $O(1 / a)$ and $M_{a}=M$ in $d=1$ and $M_{a}=a^{-1} M$ in $d=3$ is a regularizing mass (such a regularization is the one adopted in [5]). For a non-perturbative analysis we find convenient to integrate out the boson field getting a purely fermionic theory, that is

$$
\int P(d \bar{A}) e^{e \int d \mathbf{x} \bar{A}_{\mu} j_{\mu}}=e^{e^{2} \int d \mathbf{x} d \mathbf{y} v(\mathbf{x}-\mathbf{y}) j_{\mu}(\mathbf{x}) j_{\mu}(\mathbf{y})}
$$

The lattice model we consider is therefore defined by the following generating function

$$
e^{W\left(A_{\mu}, A_{\mu}^{5}, \phi\right)}=\int P(d \psi) e^{V\left(\psi, A_{\mu}, A_{\mu}^{5}, \phi\right)}
$$

where $\psi_{\mathbf{x}, i}^{ \pm}$(in $d=3 i=1,2$ while in $d=1 i=1$ and $\psi_{\mathbf{x}, 1}^{ \pm}=\psi_{\mathbf{x}}^{ \pm}$) is a set of Grassmann variables $\left\{\psi_{\mathbf{x}, i}^{\varepsilon}, \psi_{\mathbf{y}, j}^{\varepsilon^{\prime}}\right\}=0, \varepsilon, \varepsilon^{\prime}= \pm$ (with abuse of notation we denote the Grassmann variables with the same symbol as fields), $P(d \psi)$ is the fermionic integration with propagator $(2.9)$ and

$$
V\left(\psi, A_{\mu}, A_{\mu}^{5}, \phi\right)=\lambda \int d \mathbf{x} d \mathbf{y} v_{\mu, \nu}(\mathbf{x}, \mathbf{y}) j_{\mu, \mathbf{x}}(A) j_{\nu, \mathbf{y}}(A)+\nu N+B(\psi, A)+\int d \mathbf{x} A_{\mu, \mathbf{x}}^{5} j_{\mu, \mathbf{x}}^{5}(A)
$$

where the first term is the interaction, $j_{\mu}=\left(\rho, j_{1}, \ldots, j_{d}\right)$ are the lattice density and current expressed in terms of Grassmann variables, $j_{\mu}(A)$ is obtained by $j_{\mu}$ by the Perierls substitution, $\int d \mathbf{x}$ is a notation for $\int d x_{0} \sum_{x}, \lambda=e^{2}$ is the coupling and the second term is a counterterm to fix the singularity of the propagator, $N=\int d \mathbf{x} \psi_{\mathbf{x}}^{+} \psi_{\mathbf{x}}^{-}$in $d=1$ or $N=\int d \mathbf{x}\left(\psi_{1, \mathbf{x}}^{+} \psi_{1, \mathbf{x}}^{-}-\psi_{2, \mathbf{x}}^{+} \psi_{2, \mathbf{x}}^{-}\right)$in $d=3$. Finally $A_{\mu}, A_{\mu}^{5}, \phi$ are external fields ( $\phi$ is a Grassman variable) and derivatives of $W$ with respect to $A_{\mu}, A_{\mu}^{5}, \phi$ give the correlations of the current, chiral current or fermionic field respectively. In order to ensure gauge invariance for the external field $A_{\mu}$ (see (2.25) below) we define

$$
B(\psi, A)=\int d \mathbf{x} A_{0, \mathbf{x}} \rho_{\mathbf{x}}-\left(H_{0}(A)-H_{0}(0)\right)+\sum_{\varepsilon= \pm} \int d \mathbf{x} \psi_{\mathbf{x}}^{\varepsilon} \phi_{\mathbf{x}}^{-\varepsilon}
$$

with $H_{0}(A)$ given by (2.13) with Grassmann variables replacing fields and $j_{\mu, \mathbf{x}}^{5}(A)$ is obtained by $j_{\mu, \mathbf{x}}^{5}$ by the Peierls substitution; in particular the gauge invariant chiral density is

$$
\rho_{\mathbf{x}}^{5}(A)=\mathcal{Z}_{0}^{5} \frac{1}{\sin \zeta}\left(\psi_{\mathbf{x}}^{+} e^{i \int_{x_{3}}^{x_{3}+a} d s A_{3}(s)} \psi_{\mathbf{x}+a e_{3}}^{-}-\psi_{\mathbf{x}-a e_{3}}^{+} e^{i \int_{x_{3}-a}^{x_{3}} d s A_{3}(s)} \psi_{\mathbf{x}}^{-}\right)
$$

with $A_{3}(s)=A_{3}\left(x_{0}, x_{1}, x_{2}, s\right)$ and $\mathcal{Z}_{0}^{5}$ is a renormalization to be properly fixed, see below; a similar expression holds for the axial current $j_{i, \mathbf{x}}^{5}(A)$, and $\mathcal{Z}_{i}^{5}$ are the corresponding renormalizations. 
The correlations are obtained by differentiating the generating function with respect to the external fields; in particular

$$
\widehat{G}_{2}(\mathbf{k})=\left.\frac{\partial^{2} W}{\partial \widehat{\phi}_{\mathbf{k}}^{-} \partial \widehat{\phi}_{\mathbf{k}}^{+}}\right|_{0} \quad \widehat{G}_{2,1}(\mathbf{p}, \mathbf{k})=\left.\frac{\partial^{3} W}{\partial \widehat{A}_{\mu, \mathbf{p}} \partial \widehat{\phi}_{\mathbf{k}}^{-} \partial \widehat{\phi}_{\mathbf{k}+\mathbf{p}}^{+}}\right|_{0} \quad \widehat{G}_{2,1}^{5}(\mathbf{p}, \mathbf{k})=\left.\frac{\partial^{3} W}{\partial \widehat{A}_{\mu, \mathbf{p}}^{5} \partial \widehat{\phi}_{\mathbf{k}}^{-} \partial \widehat{\phi}_{\mathbf{k}+\mathbf{p}}^{+}}\right|_{0}
$$

where given a function $f\left(A_{\mu}, A_{\mu}^{5}, \phi\right)$ we denote $\left.f\left(A_{\mu}, A_{\mu}^{5}, \phi\right)\right|_{0}=f(0,0,0)$ and $\widehat{A}_{\mu}$ is the Fourier transform of $A_{\mu}$.

We define in $d=1 \widehat{G}_{\omega}(\mathbf{k}-\omega \bar{\zeta} / a)=\chi_{\omega}(\mathbf{k}) \widehat{G}_{2}(\mathbf{k})$ and in $d=3$

$$
\widehat{G}_{i, j ; \omega}(\mathbf{k}-\omega \bar{\zeta} / a)=\delta_{i, j, \omega} \chi_{\omega}(\mathbf{k}) \widehat{G}_{2, i, j}(\mathbf{k})
$$

Similarly we introduce the current correlations

$$
\begin{aligned}
\widehat{\Gamma}_{\mu, \mu_{1}, \ldots, \mu_{n}}\left(\mathbf{p}_{1}, \ldots, \mathbf{p}_{n}\right) & =\left.\frac{\partial^{n+1} W}{\partial \widehat{A}_{\mu, \mathbf{p}} \partial \widehat{A}_{\mu_{1}, \mathbf{p}_{1}} \ldots \partial \widehat{A}_{\mu_{n}, \mathbf{p}_{n}}}\right|_{0} \\
\widehat{\Gamma}_{\mu, \mu_{1}, \ldots, \mu_{n}}^{5}\left(\mathbf{p}_{1}, \ldots, \mathbf{p}_{n}\right) & =\left.\frac{\partial^{n+1} W}{\partial \widehat{A}_{\mu, \mathbf{p}}^{5} \partial \widehat{A}_{\mu_{1}, \mathbf{p}_{1}} \ldots \partial \widehat{A}_{\mu_{n}, \mathbf{p}_{n}}}\right|_{0}
\end{aligned}
$$

By Feynman graph expansion one can see that the correlations of (2.17) coincide in the formal limit in which regularizations are removed $a \rightarrow 0, M \rightarrow 0$ with massless QED in the Feynman gauge. The lattice breaks the Lorentz symmetry, so that the parameters $t, t^{\prime}$ have to be chosen as function of the coupling $\lambda$ to fix the light velocity equal to $c=1 ; \nu$ is a counterterm to fix the position of the singularity. The chiral symmetry is also broken and one has to fix the constants $\mathcal{Z}_{\mu}^{5}$ in order to ensure the following condition, if $\mathbf{k}=\mathbf{q}+\omega \bar{\zeta} / a$, $\mathbf{q}, \mathbf{p}$ small, $\omega= \pm$

$$
\widehat{G}_{2,1, \mu}(\mathbf{p}, \mathbf{k})=\omega \widehat{G}_{2,1, \mu}^{5}(\mathbf{p}, \mathbf{k})(1+O(a \mathbf{q}, a(\mathbf{q}+\mathbf{p}))
$$

The $\mathrm{AB}$ non-renormalization means that the anomaly acquires no corrections provided that the normalizations are fixed so that (2.24) holds, see e.g. [6].

While the lattice breaks chiral and Lorentz symmetry (which are only emergent), our model respects exactly gauge symmetry, as by construction

$$
W\left(A_{\mu}, A_{\mu}^{5}, \phi\right)=W\left(A_{\mu}+\partial_{\mu} \alpha_{\mathbf{x}}, A_{\mu}^{5}, e^{i \alpha_{\mathbf{x}}} \phi_{\mathbf{x}}\right)
$$

and from this we get the following Ward Identity expressing the conservation of the current

$$
\mathbf{p}_{\mu} \widehat{\Gamma}_{\mu, \mu_{1}, \ldots, \mu_{n}}=0
$$

and the relation

$$
-i \mathbf{p}_{\mu} \widehat{G}_{2,1, \mu}(\mathbf{p}, \mathbf{k})=\widehat{G}_{2}(\mathbf{k})-\widehat{G}_{2}(\mathbf{k}+\mathbf{p})
$$

The chiral symmetry is broken by the lattice so that the analogue of (2.26) for the chiral current is not true. In the emergent continuum theory the chiral symmetry holds exactly but nevertheless $\mathbf{p}_{\mu} \widehat{\Gamma}_{\mu, \mu_{1}, \ldots, \mu_{n}}^{5}$ is non vanishing, what is precisely the quantum anomaly [4]. In [11] it was shown that, in the non-interacting case, one has in the lattice theory $\mathbf{p}_{\mu} \widehat{\Gamma}_{\mu, \nu}^{5}(\mathbf{p})=\frac{1}{\pi} \varepsilon_{\mu, \nu} \mathbf{p}_{\mu}$ in $d=1$ and $\mathbf{p}_{\mu} \widehat{\Gamma}_{\mu, \nu, \sigma}^{5}\left(\mathbf{p}_{1}, \mathbf{p}_{2}\right)=\frac{1}{2 \pi^{2}} \mathbf{p}_{1, \alpha} \mathbf{p}_{2, \beta} \varepsilon_{\alpha, \beta, \nu, \sigma}$ in $d=3$, that is one gets the same result as the continuum theory. We investigate what happens to the anomaly in presence of interaction with a finite lattice. 
Theorem. For small $\lambda$ and suitable $\nu, t, t^{\prime}$ and $\mathcal{Z}_{\mu}^{5}$ chosen so that (2.24) holds, the correlations of (2.17) are, respectively for $d=1$ and $d=3$

$$
\widehat{G}_{\omega}(\mathbf{q})=\frac{|\mathbf{k}|^{\eta}}{Z} g_{\omega}^{D}(\mathbf{q})(1+R(\mathbf{q})) \quad \widehat{G}_{\omega}(\mathbf{q})=\frac{1}{Z} g_{\omega}^{D}(\mathbf{q})(1+R(\mathbf{q}))
$$

where $\eta=a \lambda^{2}+O\left(\lambda^{3}\right), Z=1+O(\lambda)$ and $R(\mathbf{q})$ non vanishing and $|R(\mathbf{q})| \leq C a|\mathbf{q}|$; moreover, up to higher order terms in $\mathbf{p}$

$$
\mathbf{p}_{\mu} \widehat{\Gamma}_{\mu, \nu}^{5}(\mathbf{p})=\frac{1}{\pi} \varepsilon_{\mu, \nu} \mathbf{p}_{\mu} \quad \mathbf{p}_{\mu} \widehat{\Gamma}_{\mu, \nu, \sigma}^{5}\left(\mathbf{p}_{1}, \mathbf{p}_{2}\right)=\frac{1}{2 \pi^{2}} \mathbf{p}_{1, \alpha} \mathbf{p}_{2, \beta} \varepsilon_{\alpha, \beta, \nu, \sigma}
$$

The above result is an emergent Adler-Bardeen theorem, as (2.29) says that there are no interaction corrections to the anomaly, even in presence of a finite lattice; its value coincides with the one of non interacting Dirac fermions. In contrast symmetry breaking terms produce non vanishing corrections to the correlations, see (2.28). The above result is rigorous, as the presence of the lattice allows to get a full non-perturbative control on the functional integrals.

In the rest of the paper a proof of the above result is provided. In section 3 we describe the Renormalization Group analysis for the lattice model (2.17), and we get the main regularity properties for the kernels of the effective potential. In section 4 we get the anomaly non-renormalization in the $d=3$ case, and in section 5 in the $d=1$ case; finally section 6 is devoted to conclusions.

\section{Renormalization Group}

As we are interested in the possible breaking of the $\mathrm{AB}$ property due the irrelevant terms, one needs an exact RG analysis in order to take them fully into account $[17,18]$. The starting point is the decomposition of the propagator in higher and lower energy degrees of freedom, that is

$$
g(\mathbf{x})=g^{(N)}(\mathbf{x})+g^{(\leq N-1)}(\mathbf{x})
$$

where $\widehat{g}^{(N)}(\mathbf{k})$ and $\widehat{g}^{(\leq N-1)}(\mathbf{k})$ are equal to $\widehat{g}(\mathbf{k})$ times $f_{N}(\mathbf{k})$ and $\chi_{N-1}(\mathbf{k})$ respectively, where $\chi_{N-1}(\mathbf{k})$ is a compact support function selecting momenta such that $|\mathbf{k}-\omega \bar{\zeta} / a| \leq \gamma^{N}$ with $\gamma>1, \gamma^{N}=1 /(10 a)$ and $f_{N}=1-\chi_{N-1}$. We can use the decomposition property $P(d \psi)=P\left(d \psi^{(\leq N-1)}\right) P\left(d \psi^{(N)}\right)$, where $P\left(d \psi^{(\leq N-1)}\right)$ and $P\left(d \psi^{(N)}\right)$ have propagator $g^{(\leq N-1)}(\mathbf{x})$ and $g^{(N)}(\mathbf{x})$. The field $\psi^{(N)}$ represents the highest energy degree of freedom; its propagator $g^{(N)}(\mathbf{x})$ decays at large distances faster than any power with rate $\gamma^{N}$ and is bounded by $\gamma^{d N}$, and it can be integrated out safely. Note that $\chi_{N-1}(\mathbf{k})$ as a function of $\mathbf{k}$ has support in two disconnected regions around $\pm \bar{\zeta} / a$; we can therefore, after shifting the momenta, write

$$
g^{(\leq N-1)}(\mathbf{x})=\sum_{\omega= \pm} e^{i \omega \frac{\bar{\zeta}}{a} \mathbf{x}} g_{\omega}^{(\leq N-1)}(\mathbf{x}) \quad \psi_{\mathbf{x}}^{ \pm(\leq N-1)}=\sum_{\omega= \pm} e^{ \pm i \omega \frac{\bar{\zeta}}{a} \mathbf{x}} \psi_{\omega, \mathbf{x}}^{ \pm(\leq N-1)}
$$


In conclusion we get

$$
\begin{aligned}
e^{W\left(A_{\mu}, A_{\mu}^{5}, \phi\right)} & =\int P\left(d \psi^{(\leq N-1)}\right) P\left(d \psi^{(N)}\right) e^{V^{(N)}\left(\psi^{(\leq N-1)}+\psi^{(N)}, \phi, A_{\mu}^{5}, A_{\mu}\right)} \\
& =\int P\left(d \psi^{(\leq N-1)}\right) e^{V^{(N-1)}\left(\psi^{(\leq N-1)}, \phi, A_{\mu}^{5}, A_{\mu}\right)}
\end{aligned}
$$

with $V^{(N-1)}\left(\psi^{(\leq N-1)}, \phi, A_{\mu}^{5}, A_{\mu}\right)$ equal to $\sum_{n=0}^{\infty} \frac{1}{n !} E_{N}^{T}(V ; n)$ and $E_{N}^{T}$ is the truncated expectation, that is the sum of connected Feynman graphs. The effective potential $V^{(N-1)}$ is given by

$$
V^{(N-1)}=\sum_{l, m} \int d \underline{\mathbf{x}} d \underline{\mathbf{y}} W_{l, m}^{(N-1)}(\underline{\mathbf{x}}, \underline{\mathbf{y}})\left[\prod_{i=1}^{l} \psi_{j_{i} \mathbf{x}_{i}, \omega_{i}}^{\varepsilon_{i}(\leq N-1)}\right]\left[\prod_{i=1}^{m} A_{\mu, \mathbf{y}_{i}}^{\sigma_{i}}\right]
$$

where $\underline{\mathbf{x}}=\mathbf{x}_{1}, \ldots, \mathbf{x}_{l}, \underline{\mathbf{y}}=\mathbf{y}_{1}, \ldots, \mathbf{y}_{m}, j=1,2$ in $d=3$ or $j=1$ in $d=1, \varepsilon_{i}= \pm, \mu=0,1$ in $d=1$ and $\mu=0,1,2,3$ in $d=3, \omega= \pm$ and $\sigma=0,5\left(A_{\mu, \mathbf{y}}^{0} \equiv A_{\mu, \mathbf{y}}\right)$.

Note that the RG integration step has two effects; the first is that the potential is now expressed as sum over monomials of fields of every order and the second that the field is splitted in two components labeled by $\omega= \pm$. The kernels $W_{l, m}^{(N-1)}$ are expressed by convergent series in $\lambda$; this follows from the representation $g^{(N)}(\mathbf{x}-\mathbf{y})=\left(f_{\mathbf{x}}, g_{\mathbf{y}}\right)$ where $($,$) is a suitable scalar product and the fact that fermionic expectation can be$ written as the determinant of a Gram matrix $M$ with elements $\left(f_{\mathbf{x}_{i}}, g_{\mathbf{x}_{j}}\right)$ with bound $|\operatorname{det} M| \leq \prod\left\|f_{\mathbf{x}_{i}}\left|\left\|\mid g_{\mathbf{x}_{i}}\right\|\right.\right.$; see e.g. [19] or [12].

We integrate the lower degrees of freedom writing $g_{\omega}^{(\leq N-1)}=\sum_{h=-\infty}^{N-1} g_{\omega}^{(h)}$ where $g_{\omega}^{(h)}$ has cut-off function $f_{h}$ with support in $\gamma^{h-1} \leq|\mathbf{k} \mp \bar{\zeta} / a| \leq \gamma^{h+1}$; by integrating the fields $\psi_{\omega}^{(N-1)}, \psi_{\omega}^{(N-2)}, \ldots, \psi_{\omega}^{(h)}$ we get an expression similar to $(3.3)$ with $P\left(d \psi^{(\leq h)}\right)$ with propagator

$$
g_{\omega}^{(\leq h)}(\mathbf{x})=\int \frac{d \mathbf{k}}{(2 \pi)^{d+1}} \frac{e^{i \mathbf{k x}}}{Z_{h}} \frac{\chi_{h}(\mathbf{k})}{-i \alpha_{\mu, \omega, h} k_{\mu}}+r_{\omega}^{h}(\mathbf{x})
$$

where $\chi_{h}=\sum_{k=-\infty}^{h} f_{k}$ and in $d=1$ one has $\alpha_{0, \omega, h}=1, \alpha_{1, \omega, h}=-i \omega v_{h}$ and in $d=3$ one has $\alpha_{0, \omega, h}=1, \quad \alpha_{1, \omega, h}=-i \sigma_{1} v_{h, 1}, \quad \alpha_{2, \omega, h}=-i \sigma_{2} v_{h, 1}, \quad \alpha_{3, \omega, h}=-i \omega \sigma_{3} v_{h, 3}$; the first term is bounded by $\gamma^{d h}$ and decays faster than any power in $\gamma^{h}|\mathbf{x}|$, while the second is smaller, being bounded by $\gamma^{d h} a \gamma^{h}$. The velocities are such that $v_{h} \rightarrow v_{-\infty}=v_{0}+O(\lambda)$ and we can tune the parameters such that $v_{-\infty}=1$. We call $\alpha_{\mu, \omega}$ simply $\alpha_{\mu, \omega, h}$ with $h=-\infty$. With this choice the first term in the r.h.s. of (3.5) is the relativistic propagator at scale $h$.

The effective potential $V^{h}$ can be decomposed in an irrelevant part, containing all the monomials with negative scaling dimension $D=(d+1)-d n / 2-m$, and a relevant and marginal part $D \geq 0$. The marginal term linear in $A$ have the form

$$
\sum_{\omega= \pm} \int \frac{d \mathbf{p}}{(2 \pi)^{d+1}}\left[Z_{\mu, h} \widehat{A}_{\mu, \mathbf{p}} \widehat{\jmath}_{\mu, \omega, \mathbf{p}}+Z_{\mu, h}^{5} \widehat{A}_{\mu, \mathbf{p}}^{5} \widehat{J}_{\mu, \omega, \mathbf{p}}^{5}\right]
$$

with (in $\left.d=3 \widehat{\psi}=\left(\widehat{\psi}_{1}, \widehat{\psi}_{2}\right)\right)$

$$
\widehat{j}_{\mu, \omega, \mathbf{p}}=\int \frac{d \mathbf{k}}{(2 \pi)^{d+1}} \widehat{\psi}_{\omega, \mathbf{k}+\mathbf{p}}^{+} \alpha_{\mu, \omega} \widehat{\psi}_{\omega, \mathbf{k}}^{-} \quad \widehat{j}_{\mu, \omega, \mathbf{p}}^{5}=\mathcal{Z}_{\mu}^{5} \int \frac{d \mathbf{k}}{(2 \pi)^{d+1}} \widehat{\psi}_{\omega, \mathbf{k}+\mathbf{p}}^{+} \alpha_{\mu, \omega}^{5} \widehat{\psi}_{\omega, \mathbf{k}}^{-} \quad \alpha_{\mu, \omega}^{5}=\omega \alpha_{\mu, \omega}
$$


The factors $Z_{\mu, h}$ or $Z_{\mu, h}^{5}$ are the renormalizations of the current and axial current respectively. The relevant term is $\gamma^{h} \nu_{h} \sum_{\omega} \int d \mathbf{x} \psi_{\mathbf{x}, \omega}^{+} \bar{\alpha} \psi_{\mathbf{x}, \omega}^{-}$with $\bar{\alpha}=1$ in $d=1$ and $\sigma_{3}$ in $d=3$ and $\nu$ has to fixed so that so that $\nu_{h}=O\left(\gamma^{h-N}\right)$. Finally in $d=1$ there is a marginal interaction

$$
\lambda_{h} \int d \mathbf{x} \psi_{\mathbf{x},+}^{+} \psi_{\mathbf{x},+}^{-} \psi_{\mathbf{x},-}^{+} \psi_{\mathbf{x},-}^{-}
$$

which is absent in $d=3$.

The kernels $W_{l, m}^{(h)}$ are obtained, see e.g. [12], by contracting the effective potentials at previous scales, and one can distinguish the contributions $W_{a, l, m}^{(h)}$, obtained contracting only marginal terms, from the contributions $W_{b, l, m}^{(h)}$ obtained contracting at least an irrelevant or relevant $\nu$ term; the series expansion are convergent and the following bound holds [12]

$$
\int d\left(\underline{\mathbf{x}} / \mathbf{x}_{1}\right)\left|W_{i, l, m}^{(h)}(\underline{\mathbf{x}})\right| \leq C \gamma^{D h} \gamma^{-\theta_{i}(N-h)} \quad \theta_{a}=0, \theta_{b}=1 \quad D=(d+1)-d l / 2-m
$$

Note that there is an essential difference between the $d=3$ and $d=1$ case; in the first case to $W_{a, n, m}^{(h)}$ no vertices with more than two fermionic lines contribute, while in the second also the local vertices quartic in $\psi$ contribute.

The flow of the running coupling constants and renormalizations is quite different. In the $d=3$ case $[20,21]$ the terms with more than 2 fields have negative dimension so that

$$
\begin{aligned}
& \frac{Z_{h-1}}{Z_{h}}=1+O\left(\lambda \gamma^{h-N}\right) \quad v_{h-1}=v_{h}+O\left(\lambda \gamma^{h-N}\right) \\
& \frac{Z_{\mu, h-1}}{Z_{\mu, h}}=1+O\left(\lambda \gamma^{h-N}\right) \quad \frac{Z_{\mu, h-1}^{5}}{Z_{\mu, h}^{5}}=1+O\left(\lambda \gamma^{h-N}\right)
\end{aligned}
$$

by (3.9). We choose the parameters so that $v_{h}=1+O\left(\lambda \gamma^{h-N}\right)$.Defining $Z_{\mu,-\infty} \equiv Z_{\mu}$, $Z_{\mu,-\infty}^{5} \equiv Z_{\mu}^{5}, Z_{-\infty} \equiv Z$ we can write

$$
Z_{\mu, h}=Z_{\mu}+O\left(\lambda \gamma^{h-N}\right)
$$

and similar expressions for $Z_{h}, Z_{\mu, h}$.

In the $d=1$ case [22-24] in contrast the interaction is marginal and the beta function of the renormalizations is given by

$$
\frac{Z_{h-1}}{Z_{h}}=1+a \lambda_{h}^{2}+O\left(\lambda_{h}^{3}\right)
$$

and similar expressions holds for $Z_{\mu, h}$ and $Z_{\mu, h}^{5}$. It turns out that, as a consequence of the emerging chiral symmetry, the beta function for $\lambda_{h}$ is asymptotically vanishing $\lambda_{h-1}=$ $\lambda_{h}+O\left(\lambda^{2} \gamma^{h-N}\right)$ and the same is true for the velocity. Note that, as $\lambda_{h} \rightarrow \lambda_{-\infty}=\lambda+O\left(\lambda^{2}\right)$, then the renormalization can be singular as $h \rightarrow-\infty$; in particular

$$
Z_{h} \sim \gamma^{\eta(h-N)}
$$

with $\eta=-a \lambda^{2}+O\left(\lambda^{3}\right)$.

The conclusion of the above analysis is that, if we suitable fix the velocities $v_{0}$ and the counterterms $\nu$ one gets (2.28), that is Lorentz invariance emerges up to corrections which are small if $\mathbf{q}$ is far from the lattice scale. 


\section{Anomaly non-renormalization; the irrelevant case}

In $d=3$ the interaction is irrelevant and, by (3.9), for $\mathbf{k} \sim \omega \bar{\zeta} / a, \mathbf{p} \sim 0, \omega= \pm \widehat{G}_{2}(\mathbf{k})=$ $\frac{1}{Z} g(\mathbf{k})(1+O(a \mathbf{q}))$ and

$$
\begin{aligned}
& \widehat{G}_{2,1, \mu}(\mathbf{p}, \mathbf{k})=Z_{\mu} \widehat{G}_{2}(\mathbf{k}) \alpha_{\mu, \omega} \widehat{G}_{2}(\mathbf{k}+\mathbf{p})(1+R) \\
& \widehat{G}_{2,1, \mu}^{5}(\mathbf{p}, \mathbf{k})=\omega \mathcal{Z}_{\mu}^{5} Z_{\mu}^{5} \widehat{G}_{2}(\mathbf{k}) \alpha_{\mu, \omega} \widehat{G}_{2}(\mathbf{k}+\mathbf{p})(1+R)
\end{aligned}
$$

with $|R| \leq C a(|\mathbf{q}|,|\mathbf{q}+\mathbf{p}|)$. Note the perfect proportionality of the vertex function to $Z_{\mu}, Z_{\mu}^{5}$ which is not true in the marginal case (the $R$ term is not subdominant). We know from the previous section that $Z, Z_{\mu}, Z_{\mu}^{5}$ are expressed by convergent series depending on all details at the lattice scale; the Ward Identity (2.27) implies the exact relation

$$
\frac{Z_{\mu}}{Z}=1
$$

A similar identity is not true for $Z_{\mu}^{5}$ and generically $Z_{\mu}^{5} / Z_{\mu}$ is a non trivial function of $\lambda$. Therefore in order to ensure the validity of (2.24) we choose

$$
\mathcal{Z}_{\mu}^{5}=\frac{Z_{\mu}}{Z_{\mu}^{5}}
$$

The anomaly coefficient is expressed in terms of

$$
\widehat{\Gamma}_{\mu, \mu_{1}, \mu_{3}}^{5}\left(\mathbf{p}_{1}, \mathbf{p}_{2}\right)=\sum_{h=-\infty}^{N} \widehat{W}_{0,3}^{(h)}\left(\mathbf{p}_{1}, \mathbf{p}_{2}\right)
$$

By (3.9) it is bounded by

$$
\left|\widehat{\Gamma}_{\mu, \mu_{1}, \mu_{3}}^{5}\left(\mathbf{p}_{1}, \mathbf{p}_{2}\right)\right| \leq C \sum_{h=-\infty}^{N} \gamma^{h}<\infty
$$

so that it is continuous as a function of $\mathbf{p}_{1}, \mathbf{p}_{2}$; it is however not differentiable as each derivative produces an extra $\gamma^{-h}$. The continuity combined with Ward Identites (2.25) are sufficient to prove that $\Gamma_{\mu, \mu_{1}, \mu_{2}}^{5}(0,0)=0$ without any explicit computation: it is sufficient to write from (2.25) $\mathbf{p}_{1, \mu_{1}} \widehat{\Gamma}_{\mu, \mu_{1}, \mu_{2}}^{5}\left(\mathbf{p}_{1}, \mathbf{p}_{2}\right)=0$ at $\mathbf{p}_{1,1}=\bar{p}_{1}$ and zero otherwise and use continuity. One would be tempted to iterate this argument for the derivative of $\widehat{\Gamma}_{\mu, \mu_{1}, \mu_{2}}^{5}$, but that is impossibile for the lack of differentiability, and indeed $\widehat{\Gamma}_{\mu, \mu_{1}, \mu_{2}}^{5}$ has non vanishing derivatives.

Regularity properties are a very efficient tool to get information on the property of the anomalies, once that $\widehat{\Gamma}_{\mu, \mu_{1}, \mu_{2}}^{5}\left(\mathbf{p}_{1}, \mathbf{p}_{2}\right)$ is suitable decomposed in order to get advantage from the dimensional gain in (3.9). We write, $\mathbf{p}=\mathbf{p}_{1}+\mathbf{p}_{2}$

$$
\widehat{\Gamma}_{\mu, \nu, \sigma}^{5}\left(\mathbf{p}_{1}, \mathbf{p}_{2}\right)=\left\langle\widehat{\jmath}_{\mu, \mathbf{p}}^{5} ; \widetilde{\jmath}_{\nu, \mathbf{p}_{1}} ; \widetilde{\jmath}_{\sigma, \mathbf{p}_{2}}\right\rangle+\Delta\left(\mathbf{p}_{1}, \mathbf{p}_{2}\right)
$$

where $\Delta$ is the Schwinger term and $\widetilde{j}$ the interacting current (obtained by the derivative in A). $\Delta$ has the same bound as the terms with $m=2,1$ hence they are differentiable. 


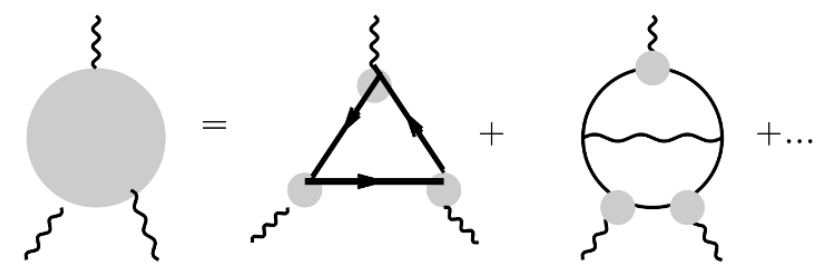

Figure 1. The decomposition of $\left\langle\widehat{\jmath}_{\mu, \mathbf{p}}^{5} ; \widetilde{\jmath}_{\nu, \mathbf{p}_{1}} ; \widetilde{\jmath}_{\sigma, \mathbf{p}_{2}}\right\rangle$.

In absence of interaction $\lambda=0\left\langle\widehat{\jmath}_{\mu, \mathbf{p}}^{5} ; \widetilde{\jmath}_{\nu, \mathbf{p}_{1}} ; \widetilde{\jmath}_{\sigma, \mathbf{p}_{2}}\right\rangle$ is expressed by the triangle graph. In presence of interaction, the RG analysis of the previous section says that

$$
\left\langle\widehat{\jmath}_{\mu, \mathbf{p}}^{5} ; \widetilde{\jmath}_{\nu, \mathbf{p}_{1}} ; \widetilde{\jmath}_{\sigma, \mathbf{p}_{2}}\right\rangle=\sum_{h=-\infty}^{N} \widehat{W}_{a, 0,3}^{(h)}+\sum_{h=-\infty}^{N} \widehat{W}_{b, 0,3}^{(h)}
$$

where the first term, containing only marginal source terms, is the triangle graph with propagators $g^{(h)} / Z_{h}$ and vertices associated to $Z_{\mu, h}, Z_{\mu, h}^{5}$, while the second is a series of terms with an arbitrary number of quartic interactions, see figure 1 . According to the bound (3.9) we have

$$
\sum_{h=-\infty}^{N}\left|\partial \widehat{W}_{b, 0,3}^{(h)}\right| \leq \sum_{h=-\infty}^{N} \gamma^{(h-N)} \leq C
$$

so that $\widehat{W}_{b, 0,3}^{(h)}$ is differentiable while $\widehat{W}_{a, 0,3}^{(h)}$ is not.

We can replace in the renormalized triangle graph the values of $Z_{\mu, h}, Z_{\mu, h}^{5}, v_{h}$ with their limiting value; the difference has again an extra $O\left(\gamma^{h-N}\right)$ so gives a differentiable contribution. Summing over the scale $h$ has the effect that the cut-off $f_{h}$ of single scale propagators add up to $\chi=\sum_{h=-\infty}^{N} f_{h}$ so that we get at the end

$$
\sum_{h=-\infty}^{N} W_{a, 0,3}^{(h)}=\frac{\mathcal{Z}_{\mu}^{5} Z_{\mu}^{5} Z_{\nu} Z_{\sigma}}{Z^{3}} I_{\mu, \nu, \sigma}\left(\mathbf{p}_{1}, \mathbf{p}_{2}\right)+G\left(\mathbf{p}_{1}, \mathbf{p}_{2}\right)
$$

where the second term is differentiable while $I_{\mu, \nu, \sigma}\left(\mathbf{p}_{1}, \mathbf{p}_{2}\right)$ is the relativistic triangle graph with propagators $\frac{\chi(\mathbf{k})}{-i \mathfrak{k}}$, that is with a momentum cut-off. In conclusion

$$
\widehat{\Gamma}_{\mu, \nu, \sigma}^{5}\left(\mathbf{p}_{1}, \mathbf{p}_{2}\right)=\frac{\mathcal{Z}_{\mu}^{5} Z_{\mu}^{5} Z_{\nu} Z_{\sigma}}{Z^{3}} I_{\mu, \nu, \sigma}\left(\mathbf{p}_{1}, \mathbf{p}_{2}\right)+H_{\mu, \nu, \sigma}^{5}\left(\mathbf{p}_{1}, \mathbf{p}_{2}\right)
$$

where $H_{\mu, \nu, \sigma}^{5}$ is continuously differentiable. By (4.2), (4.3) we get

$$
\frac{\mathcal{Z}_{\mu}^{5} Z_{\mu}^{5} Z_{\nu} Z_{\sigma}}{Z^{3}}=1
$$

In addition the contribution from the first term in (4.10) can be explicitly computed, see [15], and one gets

$$
\mathbf{p}_{\mu} I_{\mu, \nu, \sigma}\left(\mathbf{p}_{1}, \mathbf{p}_{2}\right)=\frac{1}{6 \pi^{2}} \mathbf{p}_{1, \alpha} \mathbf{p}_{2, \beta} \varepsilon_{\alpha \beta \nu \sigma} \quad \mathbf{p}_{1, \nu} I_{\mu, \nu, \sigma}\left(\mathbf{p}_{1}, \mathbf{p}_{2}\right)=\frac{1}{6 \pi^{2}} \mathbf{p}_{1, \alpha} \mathbf{p}_{2, \beta} \varepsilon_{\alpha \beta \mu \sigma}
$$


up to higher order terms, cubic in the momenta; moreover $\mathbf{p}_{2, \sigma} I_{\mu, \nu, \sigma}\left(\mathbf{p}_{1}, \mathbf{p}_{2}\right)=$ $\mathbf{p}_{2, \sigma} I_{\mu, \sigma, \nu}\left(\mathbf{p}_{2}, \mathbf{p}_{1}\right)=\frac{1}{6 \pi^{2}} \mathbf{p}_{2, \alpha} \mathbf{p}_{1, \beta} \varepsilon_{\alpha, \beta, \mu, \nu}$. Note that the r.h.s. of (4.12) do not depend on the cut-off $1 / a$; moreover either the current and the chiral current are not conserved in $I_{\mu, \nu, \sigma}$ as the momentum cut-off breaks the local gauge invariance.

It remains to evaluate the second term in (4.10); it depends on all the irrelevant terms and is expressed by a complicate series so it cannot be explicitly computed; however we show now that the information that is differentiable combined with Ward Identity (2.25) is sufficient for its determination. Indeed from the WI (2.25) we get

$$
\mathbf{p}_{1, \nu} \widehat{\Gamma}_{\mu, \nu, \sigma}^{5}\left(\mathbf{p}_{1}, \mathbf{p}_{2}\right)=0
$$

We use now the decomposition (4.10) and the differentiability of $H_{\mu, \nu, \sigma}^{5}$ to expand up to first order

$$
0=\frac{1}{6 \pi^{2}} \mathbf{p}_{1, \alpha} \mathbf{p}_{2, \beta} \varepsilon_{\alpha, \beta, \mu, \sigma}+\mathbf{p}_{1, \nu} \mathbf{p}_{1, \alpha} \frac{\partial}{\partial \mathbf{p}_{1, \alpha}} H_{\mu, \nu, \sigma}^{5}(\mathbf{0}, \mathbf{0})+\mathbf{p}_{1, \nu} \mathbf{p}_{2, \beta} \frac{\partial}{\partial \mathbf{p}_{2, \beta}} H_{\mu, \nu, \sigma}^{5}(\mathbf{0}, \mathbf{0})+O\left(\mathbf{p}^{3}\right)
$$

From the above relation we get $\frac{\partial}{\partial \mathbf{p}_{1, \alpha}} H_{\mu, \nu, \sigma}^{5}(\mathbf{0}, \mathbf{0})+\frac{\partial}{\partial \mathbf{p}_{1, \nu}} H_{\mu, \alpha, \sigma}(\mathbf{0}, \mathbf{0})=0$ and

$$
\frac{1}{6 \pi^{2}} \varepsilon_{\alpha, \beta, \mu, \sigma}=-\frac{\partial}{\partial \mathbf{p}_{2, \beta}} H_{\mu, \alpha, \sigma}^{5}(\mathbf{0}, \mathbf{0})
$$

Similarly from $\mathbf{p}_{2, \sigma} \widehat{\Gamma}_{\mu, \nu, \sigma}\left(\mathbf{p}_{1}, \mathbf{p}_{2}\right)=0$ we get

$0=\frac{1}{6 \pi^{2}} \mathbf{p}_{2, \alpha} \mathbf{p}_{1, \beta} \varepsilon_{\alpha, \beta, \mu, \nu}+\mathbf{p}_{2, \sigma} \mathbf{p}_{1, \alpha} \frac{\partial}{\partial \mathbf{p}_{1, \alpha}} H_{\mu, \nu, \sigma}(\mathbf{0}, \mathbf{0})+\mathbf{p}_{2, \sigma} \mathbf{p}_{2, \beta} \frac{\partial}{\partial \mathbf{p}_{2, \beta}} H_{\mu, \nu, \sigma}(\mathbf{0}, \mathbf{0})+O\left(\mathbf{p}^{3}\right)$,

and $\frac{1}{6 \pi^{2}} \varepsilon_{\alpha, \beta, \mu, \nu}=-\frac{\partial}{\partial \mathbf{p}_{1, \beta}} H_{\mu, \nu, \alpha}(\mathbf{0}, \mathbf{0})$. Finally

$$
\begin{aligned}
H_{\mu, \nu, \sigma}\left(\mathbf{p}_{1}, \mathbf{p}_{2}\right) & =\mathbf{p}_{1, \alpha} \frac{\partial}{\partial \mathbf{p}_{1, \alpha}} H_{\mu, \nu, \sigma}(\mathbf{0}, \mathbf{0})+\mathbf{p}_{2, \beta} \frac{\partial}{\partial \mathbf{p}_{2, \beta}} H_{\mu, \nu, \sigma}(\mathbf{0}, \mathbf{0}) \\
& =-\frac{1}{6 \pi^{2}} \mathbf{p}_{1, \alpha} \varepsilon_{\sigma, \alpha, \mu, \nu}-\frac{1}{6 \pi^{2}} \mathbf{p}_{2, \beta} \varepsilon_{\nu, \beta, \mu, \sigma}
\end{aligned}
$$

so that

$$
\begin{aligned}
\mathbf{p}_{\mu} \widehat{\Gamma}_{\mu, \nu, \sigma}^{5}\left(\mathbf{p}_{1}, \mathbf{p}_{2}\right) & =\frac{1}{6 \pi^{2}}\left(\mathbf{p}_{1, \alpha} \mathbf{p}_{2, \beta} \varepsilon_{\alpha, \beta, \nu, \sigma}-\mathbf{p}_{1, \alpha} \mathbf{p}_{2, \mu} \varepsilon_{\sigma, \alpha, \mu, \nu}-\mathbf{p}_{2, \beta} \mathbf{p}_{1, \mu} \varepsilon_{\nu, \beta, \mu, \sigma}\right) \\
& =\frac{1}{2 \pi^{2}} \mathbf{p}_{1, \alpha} \mathbf{p}_{2, \beta} \varepsilon_{\alpha, \beta, \nu, \sigma}
\end{aligned}
$$

up to higher orders terms in $\mathbf{p}$. This says that the $\mathrm{AB}$ non-renormalization property holds even in presence of symmetry breaking terms. 


\section{Anomaly non-renormalization; marginal interactions}

We have derived in the previous section the $\mathrm{AB}$ non-renormalization in a case where the interaction is irrelevant; this is in contrast with the $d=3$ case with massless photons where the interaction is marginal. However we show now that even in $d=1$, where the interaction is marginal, the $\mathrm{AB}$ renormalization holds exactly. Again we can decompose

$$
\widehat{\Gamma}_{\mu, \nu}^{5}(\mathbf{p})=\widehat{\Gamma}_{\mu, \nu}^{5, a}(\mathbf{p})+\widehat{\Gamma}_{\mu, \nu}^{5, b}(\mathbf{p})
$$

where $\widehat{\Gamma}_{\mu, \nu}^{5, a}$ contains only marginal terms and $\widehat{\Gamma}_{\mu, \nu}^{5, b}$ at least an irrelevant or relevant term; therefore by (3.9) we get

$$
\left|\widehat{\Gamma}_{\mu, \nu}^{5, i}(\mathbf{p})\right| \leq \sum_{h=0}^{N} \gamma^{\theta_{i}(h-N)}
$$

with $\theta_{a}=0, \theta_{b}=1$; therefore the first term in (5.1) is not continuous while the first is continuous. $\widehat{\Gamma}_{\mu, \nu}^{5, a}(\mathbf{p})$ has a relativistic structure and we could try to follow a strategy similar to the one in the previous section. There is however a major difference; now there are marginal terms quartic in the fields, so that the first term is expressed as a series of renormalized graphs and not by a single term. As the dominant part now corresponds to an interacting theory, it seems that it cannot be explicitly computed. We can however introduce a relativistic QFT describing Dirac fermions in $d=1+1$ with a current-current non local interaction; the corresponding generating function is given by

$$
\begin{aligned}
& e^{W_{\mathrm{rel}}\left(A, A^{5}, \phi\right)} \\
& =\int P\left(d \psi^{\leq K}\right) e^{\left.\lambda_{\infty} \widetilde{Z}^{2} \int d \mathbf{x} d \mathbf{y} v(\mathbf{x}-\mathbf{y}) j_{\mu, \mathbf{x}} j_{m, \mathbf{y}}+\sum_{\mu} \widetilde{Z}_{\mu} \int d \mathbf{x} A_{\mu} j_{\mu}+\sum_{\mu} \widetilde{Z}_{\mu}^{5} \int d \mathbf{x} A_{\mu}^{5} j_{\mu}^{5}+\int d \mathbf{x}\left(\psi_{\mathbf{x}}^{+} \phi_{\mathbf{x}}^{-}+\psi_{\mathbf{x}}^{-} \psi_{\mathbf{x}}^{+}\right)\right)}
\end{aligned}
$$

where $\psi_{\mathbf{x}, \omega}^{ \pm}, \omega= \pm$ are Grassmann variables, $j_{0, \mathbf{x}}=\psi_{\mathbf{x},+}^{+} \psi_{\mathbf{x},+}^{-}+\psi_{\mathbf{x},-}^{+} \psi_{\mathbf{x},-}^{-}, j_{1, \mathbf{x}}=i\left(\psi_{\mathbf{x},+}^{+} \psi_{\mathbf{x},+}^{-}\right.$ $\left.-\psi_{\mathbf{x},-}^{+} \psi_{\mathbf{x},-}^{-}\right), P\left(d \psi^{\leq K}\right)$ has propagator, if $\omega= \pm, \widehat{g}_{\omega}^{(\leq K)}(\mathbf{k})=\frac{\chi_{K}(\mathbf{k})}{\widetilde{Z}\left(-i k_{0}+\omega k\right)}$ with $\chi_{K}(\mathbf{k})$ a cutoff function non vanishing for $|\mathbf{k}| \leq \gamma^{K}$ and $v(\mathbf{x}-\mathbf{y})$ decaying exponentially with rate $1 / a$. This theory is in a sense the regularization of the scaling limit of the previous one, and it verifies the chiral global gauge invariance (which is broken by the lattice).

The RG analysis of (5.3) is similar to the one in section 3 and we can choose the parameters $\widetilde{Z}, \widetilde{Z}_{\mu}^{5}, \widetilde{Z}_{\mu}, \lambda_{\infty}$ in (5.3) as function of $\lambda$ so that the limiting value at $h=-\infty$ of the corresponding running coupling constants is the same as in the lattice theory. By this choice the difference in the running coupling constants is $O\left(\gamma^{h-N}\right)$ so that we get the decomposition

$\widehat{\Gamma}_{\mu, \nu}^{5}(\mathbf{p})=\mathcal{Z}_{\mu}^{5}\left[\left.\frac{\partial^{2} W_{\mathrm{rel}}\left(A, A^{5}, \phi\right)}{\partial \widehat{A}_{\mu}^{5} \partial \widehat{A}_{\nu}}\right|_{0}+H_{\mu, \nu}^{5}(\mathbf{p})\right] \quad \widehat{\Gamma}_{\mu, \nu}(\mathbf{p})=\left[\left.\frac{\partial^{2} W_{\mathrm{rel}}\left(A, A^{5}, \phi\right)}{\partial \widehat{A}_{\mu} \partial \widehat{A}_{\nu}}\right|_{0}+H_{\mu, \nu}(\mathbf{p})\right]$

where $H_{\mu, \nu}(\mathbf{p}), H_{\mu, \nu}^{5}(\mathbf{p})$ continuous by (3.9); similarly, up to subdominant terms in the momentum,

$$
\widehat{G}_{2, \mu}=\frac{\partial^{2} W_{\text {rel }}}{\partial \widehat{\phi}^{+} \partial \widehat{\phi}^{-}} \quad \widehat{G}_{2,1, \mu}=\frac{\partial^{3} W_{\text {rel }}}{\partial \widehat{A}_{\mu} \partial \widehat{\phi}^{+} \partial \widehat{\phi}^{-}} \quad \widehat{G}_{2,1, \mu}^{5}=\mathcal{Z}_{\mu}^{5} \frac{\partial^{3} W_{\text {rel }}}{\partial \widehat{A}_{\mu}^{5} \partial \widehat{\phi}^{+} \partial \widehat{\phi}^{-}}
$$




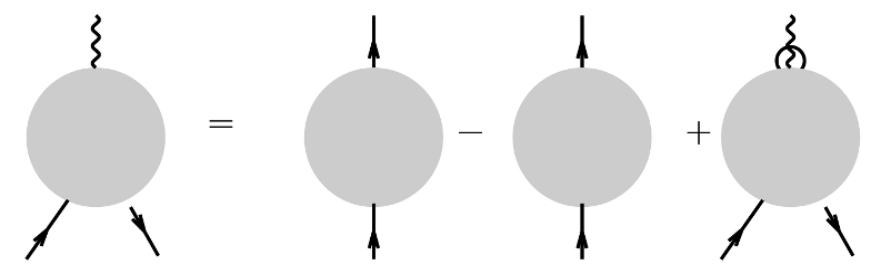

Figure 2. The WI for the vertex function of (5.3) where the last term is the extra term due to the $C$ factor.

We can take advantage from the fact that the model (5.3) verifies global and axial symmetries; however local symmetries are broken by the presence of the momentum cutoff and this produces extra anomalous terms in the WI for the global and axial current. Note indeed that, if $D_{\omega}(\mathbf{k})=-i k_{0}+\omega k$

$$
\widehat{g}_{\omega}^{(\leq K)}(\mathbf{k})-\widehat{g}_{\omega}^{(\leq K)}(\mathbf{k}+\mathbf{p})-\widehat{g}_{\omega}^{(\leq K)}(\mathbf{k}) D_{\omega}(\mathbf{p}) g_{\omega}^{(\leq K)}(\mathbf{k}+\mathbf{p})=\widehat{g}_{\omega}^{(\leq K)}(\mathbf{k}) C(\mathbf{k}, \mathbf{p}) \widehat{g}_{\omega}^{(\leq K)}(\mathbf{k}+\mathbf{p})
$$

with $C(\mathbf{k}, \mathbf{p})=D_{\omega}(\mathbf{k})\left(\chi_{K}^{-1}(\mathbf{k})-1\right)-D_{\omega}(\mathbf{k}+\mathbf{p})\left(\chi_{K}^{-1}(\mathbf{k}+\mathbf{p})-1\right)$ (the r.h.s.would be zero in absence of cut-off). The presence of this extra term produce an additional factor in the WI, see figure 2; as proven in [22-24] in the $K \rightarrow \infty$ limit the following WI for the vertex and chiral vertex are obtained

$$
\begin{aligned}
& -i p_{0} \frac{1}{\widetilde{Z}_{0}} \frac{\partial^{3} W_{\mathrm{rel}}}{\partial \widehat{A}_{0, \mathbf{p}} \partial \widehat{\phi}_{\mathbf{k}, \omega}^{-} \partial \widehat{\phi}_{\mathbf{k}+\mathbf{p}, \omega}^{+}}+\frac{p_{1}}{\widetilde{Z}_{1}} \frac{\partial^{3} W_{\mathrm{rel}}}{\partial \widehat{A}_{1, \mathbf{p}} \partial \widehat{\phi}_{\mathbf{k}, \omega}^{-} \partial \widehat{\phi}_{\mathbf{k}+\mathbf{p}, \omega}^{+}}=\frac{1}{\widetilde{Z}(1-\tau)}\left(\frac{\partial^{2} W_{\mathrm{rel}}}{\partial \widehat{\phi}_{\mathbf{k}, \omega}^{-} \partial \widehat{\phi}_{\mathbf{k}, \omega}^{+}}-\frac{\partial^{2} W_{\mathrm{rel}}}{\partial \widehat{\phi}_{\mathbf{k}+\mathbf{p}, \omega}^{-} \partial \widehat{\phi}_{\mathbf{k}+\mathbf{p}, \omega}^{+}}\right) \\
& -i p_{0} \frac{1}{\widetilde{Z}_{0}^{5}} \frac{\partial^{3} W_{\mathrm{rel}}}{\partial \widehat{A}_{0, \mathbf{p}}^{5} \partial \phi_{\mathbf{k}, \omega}^{-} \partial \phi_{\mathbf{k}+\mathbf{p}, \omega}^{+}}+\frac{p_{1}}{\widetilde{Z}_{1}^{5}} \frac{\partial^{3} W_{\mathrm{rel}}}{\partial \widehat{A}_{1, \mathbf{p}}^{5} \partial \phi_{\mathbf{k}, \omega}^{-} \partial \phi_{\mathbf{k}+\mathbf{p}, \omega}^{+}}=\frac{\omega}{\widetilde{Z}(1+\tau)}\left(\frac{\partial^{2} W_{\mathrm{rel}}}{\partial \widehat{\phi}_{\mathbf{k}, \omega}^{-} \partial \widehat{\phi}_{\mathbf{k}, \omega}^{+}}-\frac{\partial^{2} W_{\mathrm{rel}}}{\partial \widehat{\phi}_{\mathbf{k}+\mathbf{p}, \omega}^{-} \partial \widehat{\phi}_{\kappa+\mathbf{p}, \omega}^{+}}\right)
\end{aligned}
$$

and $\tau=\lambda_{\infty} / 4 \pi$. The extra term in the WI produced by the $C$ - term reduces, in the limit $K \rightarrow \infty$, to the vertex function times the constant $\tau$ (which is the graph for the anomaly in $d=1$ with momentum cut-off).

The fact that the vertex and 2-point function of (5.7) and lattice model (computed at $\mathbf{q}+\omega \zeta / a$ with $\mathbf{q}$ small) are close up to $O(a \mathbf{q})$ terms says that the first of the WI (5.7) coincides with (2.27); this imposes constraints for the parameters of effective QFT (5.3), that is

$$
\frac{\widetilde{Z}_{1}}{\widetilde{Z}_{0}}=1 \quad \frac{\widetilde{Z}_{0}}{\widetilde{Z}}=1-\tau
$$

We have now to choose $\mathcal{Z}_{\mu}^{5}$ by (2.24); from (5.7) in the limit $p_{0} \rightarrow 0, p \rightarrow 0$

$$
\widehat{G}_{2,1}^{5}=i \omega \frac{\mathcal{Z}_{0}^{5} \widetilde{Z}_{0}^{5}}{\widetilde{Z}(1+\tau)} \partial_{0} \frac{\partial^{2} W_{\mathrm{rel}}}{\partial \widehat{\phi}_{\mathbf{k}, \omega}^{-} \partial \widehat{\phi}_{\mathbf{k}, \omega}^{+}} \quad \widehat{G}_{2,1}=i \frac{\widetilde{Z}_{0}}{\widetilde{Z}(1-\tau)} \partial_{0} \frac{\partial^{2} W_{\mathrm{rel}}}{\partial \widehat{\phi}_{\mathbf{k}, \omega}^{-} \partial \widehat{\phi}_{\mathbf{k}, \omega}^{+}}
$$

and a similar expression for $\mu=1$ so that

$$
\mathcal{Z}_{i}^{5}=\frac{1+\tau}{1-\tau} \frac{\widetilde{Z}_{i}}{\widetilde{Z}_{i}^{5}}=(1+\tau) \frac{\widetilde{Z}}{\widetilde{Z}_{i}^{5}}
$$


The WI for the current correlations of (5.3) are

$$
\sum_{\mu} \mathbf{p}_{\mu} \frac{\widetilde{Z}}{\widetilde{Z}_{\mu}^{5}} \frac{\widetilde{Z}}{\widetilde{Z}_{\nu}} \frac{\partial^{2} W_{\mathrm{rel}}}{\partial \widehat{A}_{\mu}^{5} \partial \widehat{A}_{\nu}}=\frac{\varepsilon_{\mu, \nu} \mathbf{p}_{\mu}}{1+\tau} \frac{1}{2 \pi} \quad \sum_{\nu} \mathbf{p}_{\nu} \frac{\widetilde{Z}}{\widetilde{Z}_{\mu}^{5}} \frac{Z}{Z_{\nu}} \frac{\partial^{2} W_{\mathrm{rel}}}{\partial \widehat{A}_{\mu}^{5} \partial \widehat{A}_{\nu}}=\frac{\varepsilon_{\nu, \mu} \mathbf{p}_{\nu}}{1-\tau} \frac{1}{2 \pi}
$$

and from (5.8), (5.10)

$$
\frac{1}{1-\tau} \sum_{\mu} \mathbf{p}_{\mu} \mathcal{Z}_{\mu}^{5} \frac{\partial^{2} W_{\mathrm{rel}}}{\partial \widehat{A}_{\mu}^{5} \partial \widehat{A}_{\nu}}=\varepsilon_{\mu, \nu} \mathbf{p}_{\mu} \frac{1}{2 \pi} \quad \frac{1}{1+\tau} \sum_{\nu} \mathbf{p}_{\nu} \mathcal{Z}_{\mu}^{5} \frac{\partial^{2} W}{\partial \widehat{A}_{\mu}^{5} \partial \widehat{A}_{\nu}}=\varepsilon_{\rho, \mu} \mathbf{p}_{\rho} \frac{1}{2 \pi}
$$

Now we use that the lattice Ward identity (2.25) and the decomposition (5.4)

$$
\mathbf{p}_{\nu} \widehat{\Gamma}_{\mu, \nu}^{5}=\mathcal{Z}_{\mu}^{5} \sum_{\nu} \mathbf{p}_{\nu}\left[\frac{\partial^{2} W}{\partial \widehat{A}_{\mu}^{5} \partial \widehat{A}_{\nu}}+H_{\mu, \nu}\right]=0
$$

from which we get

$$
\varepsilon_{\rho, \mu} \frac{(1+\tau)}{\mathcal{Z}_{\mu}^{5}} \mathbf{p}_{\rho} \frac{1}{2 \pi}+\mathbf{p}_{\nu} H_{\mu, \nu}(\mathbf{p})=0
$$

In contrast with $\Gamma_{\mu, \nu}^{5}(\mathbf{p})$, we know that $H_{\mu, \nu}(\mathbf{p})$ is continuous in $\mathbf{p}$ so that

$$
-\varepsilon_{\rho, \mu} \frac{(1+\tau)}{\mathcal{Z}_{\mu}^{5}} \frac{1}{2 \pi}=H_{\mu, \rho}(0)
$$

and, up to higher orders in $\mathbf{p}$

$$
\begin{aligned}
\mathbf{p}_{\mu} \widehat{\Gamma}_{\mu, \nu}^{5}(\mathbf{p}) & =\sum_{\mu} \mathbf{p}_{\mu} \mathcal{Z}_{\mu}^{5}\left[\frac{\partial^{2} W_{\mathrm{rel}}}{\partial \widehat{A}_{\mu}^{5} \partial \widehat{A}_{\nu}}+H_{\mu, \nu}\right] \\
& =\varepsilon_{\mu, \nu} \frac{(1-\tau)}{2 \pi} \mathbf{p}_{\mu}-\varepsilon_{\nu, \mu} \frac{(1+\tau)}{2 \pi} \mathbf{p}_{\mu}=[(1-\tau)+(1+\tau)] \varepsilon_{\mu, \nu} \mathbf{p}_{\mu} \frac{1}{2 \pi}=\varepsilon_{\mu, \nu} \mathbf{p}_{\mu} \frac{1}{\pi}
\end{aligned}
$$

so that the factor $\tau$, depending on $\lambda$, cancels out and also in the marginal case the anomaly is non-renormalized.

\section{Conclusions}

The renormalizability of the Standard Model relies on the AB non renormalization property which is used in the anomaly cancellation. It is therefore interesting to see if the anomaly non-renormalization holds generically even when symmetry breaking terms are present at the Planck scale, or if in contrast its validity requires that they are absent or at least of special form. We have investigated such a question in QED lattice model both when the interaction is irrelevant or marginal, showing that the $\mathrm{AB}$ property holds exactly even if Lorentz or chiral symmetry is broken and corrections to correlations are present. The fact that the corrections to the anomaly are due to irrelevant terms requires the use of exact and non-perturbative RG methods. It would be interesting to establish a similar property removing the mass regularization of photons; in such a case fermionic cancellations are not sufficient to achieve convergence and large/small field decomposition is necessary to get non-perturbative results. 


\section{Acknowledgments}

This work has been supported by MIUR, PRIN 2017 project MaQuMA. PRIN201719 VMAST01.

Open Access. This article is distributed under the terms of the Creative Commons Attribution License (CC-BY 4.0), which permits any use, distribution and reproduction in any medium, provided the original author(s) and source are credited.

\section{References}

[1] A.M. Polyakov, Gauge fields and strings, Contemp. Concepts Phys. 3 (1987) 1 [InSPIRE].

[2] J. Polchinski, Effective field theory and the Fermi surface, in Recent directions in particle theory: from superstrings and blackholes to the standard model, Boulder, CO, U.S.A., 1-26 June 1992, pp. 235-274 (1992).

[3] E. Witten, Symmetry and emergence, Nature Phys. 14 (2018) 116.

[4] S.L. Adler, Axial vector vertex in spinor electrodynamics, Phys. Rev. 177 (1969) 2426 [INSPIRE].

[5] S.L. Adler and W.A. Bardeen, Absence of higher order corrections in the anomalous axial vector divergence equation, Phys. Rev. 182 (1969) 1517 [INSPIRE].

[6] S.L. Adler, Anomalies to all orders in 50 Years of Yang-Mills Theory, World Scientific, pp. 187-228 (2005) [DOI].

[7] S.R. Coleman and S.L. Glashow, High-energy tests of Lorentz invariance, Phys. Rev. D 59 (1999) 116008 [hep-ph/9812418] [INSPIRE].

[8] J. Collins, A. Perez, D. Sudarsky, L. Urrutia and H. Vucetich, Lorentz invariance and quantum gravity: an additional fine-tuning problem?, Phys. Rev. Lett. 93 (2004) 191301 [gr-qc/0403053] [INSPIRE].

[9] R. Gambini, S. Rastgoo and J. Pullin, Small Lorentz violations in quantum gravity: do they lead to unacceptably large effects?, Class. Quant. Grav. 28 (2011) 155005 [arXiv:1106.1417] [INSPIRE].

[10] J. Polchinski, Comment on [arXiv:1106.1417] 'Small Lorentz violations in quantum gravity: do they lead to unacceptably large effects?', Class. Quant. Grav. 29 (2012) 088001 [arXiv:1106.6346] [INSPIRE].

[11] H.B. Nielsen and M. Ninomiya, Adler-Bell-Jackiw anomaly and Weyl fermions in crystal, Phys. Lett. 130B (1983) 389 [INSPIRE].

[12] V. Mastropietro, Non Perturbative Renormalization, World Scientific (2008).

[13] C. Kopper and B. Leveque, Regularized path integrals and anomalies: U(1) chiral gauge theory, J. Math. Phys. 53 (2012) 022305 [arXiv:1112.3526] [InSPIRE].

[14] A. Zee, Axial vector anomalies and the scaling property of field theory, Phys. Rev. Lett. 29 (1972) 1198 [INSPIRE].

[15] A. Giuliani, V. Mastropietro and M. Porta, Anomaly non-renormalization in interacting Weyl semimetals, arXiv:1907.00682 [INSPIRE]. 
[16] A.A. Anselm and A.A. Johansen, Radiative Corrections to the Axial Anomaly, JETP Lett. 49 (1989) 214 [INSPIRE].

[17] G. Gallavotti, Renormalization theory and ultraviolet stability for scalar fields via renormalization group methods, Rev. Mod. Phys. 57 (1985) 471 [INSPIRE].

[18] J. Polchinski, Renormalization and Effective Lagrangians, Nucl. Phys. B 231 (1984) 269 [INSPIRE].

[19] K. Gawędzki and A. Kupiainen, Gross-Neveu model through convergent perturbation expansions, Commun. Math. Phys. 102 (1985) 1 [INSPIRE].

[20] V. Mastropietro, Interacting Weyl semimetals on a lattice, J. Phys. A 47 (2014) 465003 [arXiv: 1310.5638] [INSPIRE].

[21] V. Mastropietro, Weyl semimetallic phase in an interacting lattice system, J. Stat. Phys. 157 (2014) 830 [arXiv: 1405.6844] [INSPIRE].

[22] G. Benfatto, P. Falco and V. Mastropietro, Functional Integral Construction of the Thirring model: Axioms verification and massless limit, Commun. Math. Phys. 273 (2007) 67 [hep-th/0606177] [INSPIRE].

[23] G. Benfatto, P. Falco and V. Mastropietro, Massless sine-Gordon and massive Thirring models: Proof of the Coleman's equivalence, Commun. Math. Phys. 285 (2009) 713 [arXiv:0711.5010] [INSPIRE].

[24] G. Benfatto, P. Falco and V. Mastropietro, Universal Relations for Non Solvable Statistical Models, Phys. Rev. Lett. 104 (2010) 075701 [arXiv:0909.2707] [INSPIRE]. 